

\title{
Étude de l'efficacité du couplage entre la source lumineuse et le milieu actif dans un laser à pompage optique
}

\author{
Pierre Brun, Jeanine Bonamy
}

\section{To cite this version:}

Pierre Brun, Jeanine Bonamy. Étude de l'efficacité du couplage entre la source lumineuse et le milieu actif dans un laser à pompage optique. Revue de Physique Appliquée, 1967, 2 (1), pp.38-44. 10.1051/rphysap:019670020103800 . jpa-00242762

HAL Id: jpa-00242762

https://hal.science/jpa-00242762

Submitted on 1 Jan 1967

HAL is a multi-disciplinary open access archive for the deposit and dissemination of scientific research documents, whether they are published or not. The documents may come from teaching and research institutions in France or abroad, or from public or private research centers.
L'archive ouverte pluridisciplinaire HAL, est destinée au dépôt et à la diffusion de documents scientifiques de niveau recherche, publiés ou non, émanant des établissements d'enseignement et de recherche français ou étrangers, des laboratoires publics ou privés. 


\title{
ÉTUDE DE L'EFFIGAGITÉ DU GOUPLAGE ENTRE LA SOURGE LUMINEUSE ET LE MILIEU AGTIF DANS UN LASER A POMPAGE OPTIQUE
}

\author{
Par Pierre BRUN et Mme Jeanine BONAMY, \\ Faculté des Sciences de Rennes.
}

\begin{abstract}
Résumé. - Nous comparons les rendements de deux configurations géométriques utilisées dans la réalisation des lasers à pompage optique en supposant que les sources émettent selon la loi de Lambert.

La configuration elliptique est celle qui nécessite le moins d'énergie pour atteindre le seuil et qui donne le meilleur rendement.
\end{abstract}

\begin{abstract}
The efficiencies of two pumping schemes for pulsed optical masers are compared ; the lamp is assumed to be a radiator having a Lambertian radiation pattern.

The elliptical configuration requires less energy to reach threshold and gives the best energy conversion efficiency above threshold.
\end{abstract}

I. Introduction. - Parmi les diverses configurations géométriques utilisées dans la réalisation des lasers à pompage optique, on rencontre le plus fréquemment la lampe hélicoïdale coaxiale au « récepteur » et la lampe linéaire avec réflecteur à section elliptique.

Le problème consistant à transférer le maximum d'énergie fournie par la source sur le récepteur a déjà fait l'objet d'un certain nombre de travaux théoriques ou expérimentaux $[3,4,5,6]$. Nous avons repris le calcul du rendement :

$$
\eta=\frac{\text { énergie reçue par le récepteur }}{\text { énergie fournie par la source }}
$$

pour les deux configurations citées plus haut en admettant que les sources lumineuses utilisées rayonnent suivant la loi de Lambert. Il en résulte un net écart par rapport aux résultats obtenus précédemment et les vérifications expérimentales confirment nos hypothèses avec une bonne précision.

Dans toute la suite, nous supposerons que le " récepteur » est de forme cylindrique, ce qui correspond au cas le plus fréquent des cristaux utilisés dans les lasers.

Il est à noter de plus que nous calculons uniquement l'énergie totale reçue par le récepteur sans nous préoccuper de sa distribution spatiale. Dans le cas de lampes hélicoïdales, qui a d'ailleurs été très peu étudié quantitativement, le pompage du cristal se fait uniformément; dans la configuration elliptique [1], on obtient une meilleure focalisation de la lumière sur le cristal mais alors la distribution d'énergie à l'intérieur de celui-ci ne peut, bien sûr, être uniforme [2]. Il est certain dans ce cas que la condition de" rendement optimum ne correspond pas forcément à un « pompage » uniforme du milieu actif.
II. Flashes hélicoïdaux et réflecteurs cylindriques. 1. Position DU PROBlème. - Un cristal cylindrique de longueur $l$ et de rayon $r$ est situé sur l'axe d'une lampe hélicoïdale de hauteur $L$ et de rayon $R$ ( fig. 1).

Nous nous proposons de calculer le rendement de cette configuration.

Nous assimilerons la lampe à un filament hélicoïdal dont chaque élément rayonne selon la loi de Lambert. On peut en effet admettre que le diamètre du tube constituant la lampe est négligeable devant celui du cylindre hélicoïdal.

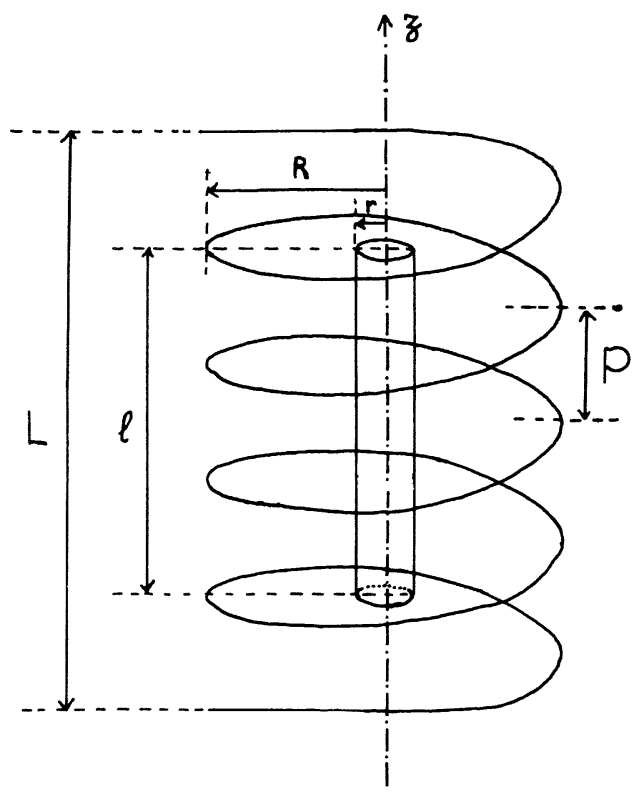

FIG. 1. - Schéma de la configuration hélicoïdale. 
Dans l'hypothèse lambertienne, sur un cône d'axe $\mathrm{d} l$ et de demi-angle au sommet $\beta$ l'intensité est la même et elle est :

$$
I=I_{0} \sin \beta
$$

$I_{0}$ étant l'intensité dans une direction perpendiculaire au filament ( fig. $2 b$ ).

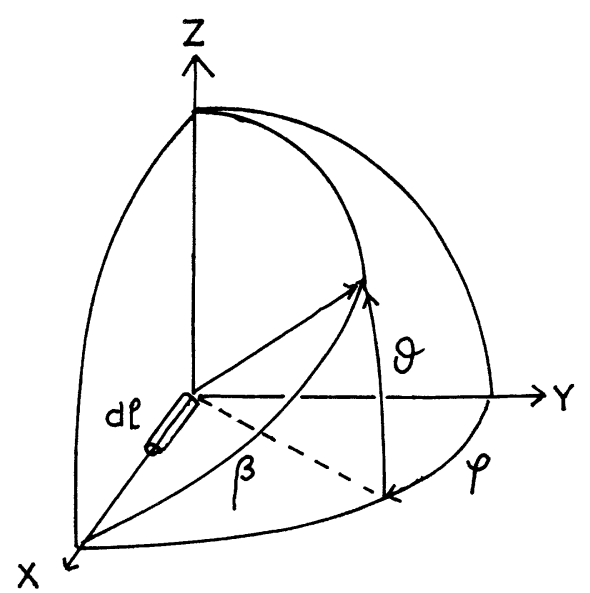

FIG. $2 a$. - Trièdre de référence

l'élément $\mathrm{d} l$ de lampe est dirigé selon l'axe $\mathrm{X}^{\prime} \mathrm{X}$.

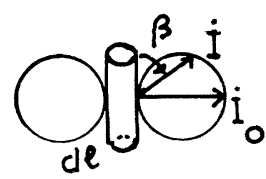

FIG. 2 b. - Émission selon la loi de Lambert dans un plan contenant l'axe de l'élément $\mathrm{d} l$ de la source.

2. RÉSOlution DU PROBLÈme. - Nous prendrons un système de coordonnées sphériques dans lequel l'élément $\mathrm{d} l$ de la lampe sera dirigé selon $\mathrm{X}^{\prime} \mathrm{X}$ ( fig. $2 a$ ). L'angle solide élémentaire $d \Omega$ est donné par

$$
\mathrm{d} \Omega=\cos \theta \mathrm{d} \theta \mathrm{d} \varphi
$$

et le flux élémentaire émis dans cet angle solide est :

$$
\begin{aligned}
\mathrm{d}[\mathrm{d} \Phi]=I \mathrm{~d} \Omega=I_{0} \frac{\cos \theta \mathrm{d} \theta \mathrm{d} \varphi}{\sqrt{1+\operatorname{tg}^{2} \varphi \cos ^{2} \theta}} \\
=I_{0} \frac{\cos \varphi \mathrm{d} \theta \mathrm{d} \varphi}{\sqrt{1+\operatorname{tg}^{2} \theta \cos ^{2} \varphi}}
\end{aligned}
$$

avec

$$
\sin \beta=1 / \sqrt{1+\operatorname{tg}^{2} \varphi \cos ^{2} \theta}
$$

Posons

$$
\operatorname{tg} \theta=k
$$

alors

$$
\mathrm{d} \Phi=I_{0} \int_{\theta_{1}}^{\theta_{2}} \mathrm{~d} \theta \int_{-\varphi_{0}}^{+\varphi_{0}} \frac{\cos \varphi \mathrm{d} \varphi}{\sqrt{1+k^{2} \cos ^{2} \varphi}}
$$

où $\theta_{1}, \theta_{2}$ et $\varphi_{0}$ sont indiqués sur la figure $3\left(I_{0}\right.$ est un élément différentiel que nous expliciterons par la suite).

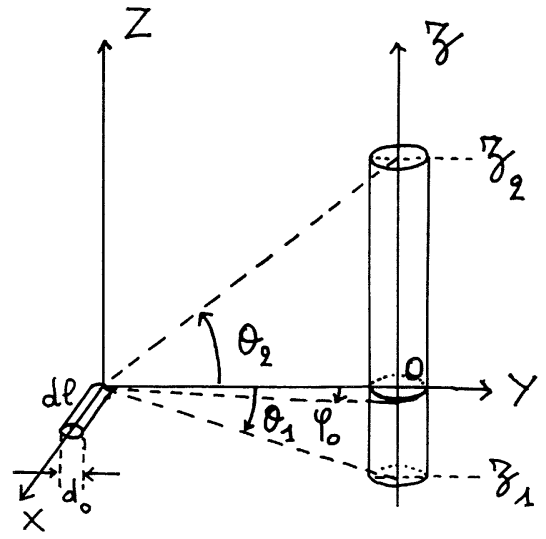

Fig. 3. - Le flux émis par la source est recueilli par le récepteur à l'intérieur des angles limites $\theta_{1}, \theta_{2}$ et $2 \varphi_{0}$.

En posant $\sin \varphi=x$, il vient

$$
\begin{aligned}
\mathrm{d} \Phi & =I_{0} \int_{\theta_{\mathrm{t}}}^{\theta_{2}} \frac{\mathrm{d} \theta}{k} \int_{-x_{0}}^{+x_{0}} \frac{\mathrm{d} x}{\sqrt{a^{2}-x^{2}}} \\
a & =\sqrt{1+k^{2}} / k=1 / \sin \varphi .
\end{aligned}
$$

En posant encore $\sin \varphi \sin \theta=u$, il vient enfin

$$
\mathrm{d} \Phi=2 I_{0} \int_{u_{1}}^{u_{2}} \frac{\arcsin u}{u} \mathrm{~d} u
$$

avec $u_{1}=\sin \varphi_{0} \sin \theta_{1} \quad-1<u_{1}<1$

$$
u_{2}=\sin \varphi_{0} \sin \theta_{2} \quad-1<u_{2}<1 .
$$

Il n'existe pas de forme analytique simple de cette intégrale. Nous utiliserons donc un développement en série

$$
\frac{\arcsin u}{u}=1+\sum_{n=1}^{\infty} C_{n} \frac{u^{2 n}}{2 n+1}
$$

avec

$$
C_{n}=\frac{1.3 .5 \ldots(2 n-1)}{2.4 .6 \ldots 2 n}
$$

Si l'on écrit

$$
J=\int_{u_{1}}^{u_{2}} \frac{\arcsin u}{u} \mathrm{~d} u
$$

on a :

$$
J=\left[u_{2}-u_{1}\right]+\sum_{n=1}^{\infty} \frac{C_{n}}{(2 n+1)^{2}}\left[u_{2}^{2 n+1}-u_{1}^{2 n+1}\right]
$$

alors

$\mathrm{d} \Phi=2 I_{0}\left\{\left[u_{2}-u_{1}\right]+\sum_{n=1}^{\infty} \frac{C_{n}}{(2 n+1)^{2}}\left[u_{2}^{2 n+1}-u_{1}^{2 n+1}\right]\right.$

Il est aisé de vérifier que la convergence rapide de la série permet de se limiter à un très petit nombre de termes. Ayant résolu ce premier problème, nous allons poursuivre le calcul par l'introduction de $z$ dans l'expression de $\mathrm{d} \Phi$. 
Nous avons dit précédemment que $I_{0}$ est différentiel. En effet, si nous prenons un petit élément de lampe de rayon $d_{0} / 2$ et de longueur $\mathrm{d} l$ ( $f g .3$ ), le flux total émis par cet élément est

$$
\pi^{2} I_{0}=E \mathrm{~d} S=E \pi d_{0} \mathrm{~d} l
$$

où $E$ est l' « irradiance » de la source, soit

$$
I_{0}=\frac{E d_{0}}{\pi} \mathrm{d} l
$$

Mais si $z$ est l'axe de l'hélice,

$$
\mathrm{d} l=\frac{\sqrt{p^{2}+R^{2}}}{p} \mathrm{~d} z
$$

où $p$ est le pas et $R$ le rayon, soit enfin

$$
\Phi=\frac{2 E d_{0} \sqrt{p^{2}+R^{2}}}{p \pi} \int_{\frac{l-L}{2}}^{\frac{l+L}{2}}\left[\int_{u_{1}}^{u_{2}} \frac{\arcsin u}{u} \mathrm{~d} u\right] \mathrm{d} z .
$$

On avait posé

$$
J=\int_{u_{1}}^{u_{2}} \frac{\arcsin u}{u} \mathrm{~d} u
$$

avec

$$
\begin{aligned}
& u_{1}=\sin \varphi_{0} \sin \theta_{1} \\
& u_{2}=\sin \varphi_{0} \sin \theta_{2}
\end{aligned}
$$

$\sin \varphi_{0}=a$ est une constante pour la suite du calcul $\sin \theta_{2}=z_{2} / \sqrt{z_{2}^{2}+R^{2}} \quad \sin \theta_{1}=-z_{1} / \sqrt{z_{1}^{2}+R^{2}}$ on a en outre $z_{2}-z_{1}=l$.

Posons $z_{2}=z$, alors $z_{1}=z-l$.

$$
\begin{aligned}
& J(z)=a\left[\frac{z}{\sqrt{z^{2}+R^{2}}}+\right.\left.\frac{z-l}{\sqrt{(z-l)^{2}+R^{2}}}\right] \\
&+\sum_{n=1}^{\infty} \frac{C_{n} a^{2 n+1}}{(2 n+1)^{2}}\left[\left(\frac{z}{\sqrt{z^{2}+R^{2}}}\right)^{2 n+1}\right. \\
&\left.+\left(\frac{z-l}{\sqrt{(z-l)^{2}+R^{2}}}\right)^{2 n+1}\right] .
\end{aligned}
$$

Il suffit maintenant de calculer l'intégrale :

$$
B=\int_{\frac{l-L}{2}}^{\frac{l+L}{2}} J(z) \mathrm{d} z .
$$

En faisant le changement de variable $z-l=x$, nous remarquons que :

$$
\int_{\frac{l-L}{2}}^{l+L} \frac{(z-l)}{\sqrt{(z-l)^{2}+R^{2}}} \mathrm{~d} z=\int_{\frac{l-L}{2}}^{\frac{l+L}{2}} \frac{x \mathrm{~d} x}{\sqrt{x^{2}+R^{2}}}
$$

d'où le résultat :

$$
\begin{aligned}
& B=2\left[a \int_{\frac{l-L}{2}}^{\frac{l+L}{2}} \frac{z \mathrm{~d} z}{\left(z^{2}+R^{2}\right)^{1 / 2}}+\frac{a^{3}}{18} \int_{\frac{l-L}{2}}^{\frac{l+L}{2}} \frac{z^{3} \mathrm{~d} z}{\left(z^{2}+R^{2}\right)^{3 / 2}}\right. \\
& \left.+\frac{3 a^{5}}{200} \int_{\frac{l-L}{2}}^{\frac{l+L}{2}} \frac{z^{5} \mathrm{~d} z}{\left(z^{2}+R^{2}\right)^{5 / 2}}+\ldots\right] \\
& B=2\left[B_{1}+B_{2}+B_{3}+\ldots\right] \\
& B_{1}=a\left[\sqrt{\left(\frac{L+l}{2}\right)^{2}+R^{2}}-\sqrt{\left(\frac{L-l}{2}\right)^{2}+R^{2}}\right] \\
& B_{2}=\frac{a^{3}}{18}\left[\sqrt{\left(\frac{L+l}{2}\right)^{2}+R^{2}}-\sqrt{\left(\frac{L-l}{2}\right)^{2}+R^{2}}\right. \\
& \left.+\frac{R^{2}}{\sqrt{\left(\frac{L+l}{2}\right)^{2}+R^{2}}}-\frac{R^{2}}{\sqrt{\left(\frac{L-l}{2}\right)^{2}+R^{2}}}\right] \\
& \Phi=\frac{2 E d_{0} \sqrt{p^{2}+R^{2}}}{p \pi} B .
\end{aligned}
$$

Revenons à la définition du rendement

$$
\begin{array}{cc} 
& \eta=\Phi / \Phi_{\text {total }} \\
\text { avec } & \Phi_{\text {tot }}=\frac{E d_{0}}{p} \sqrt{p^{2}+R^{2}} \pi L \\
\text { d'où } & \eta=2 B / \pi^{2} L .
\end{array}
$$

Intervention $d u$ réflecteur. - Jusqu'ici nous n'avons considéré que des rayons émis dans l'angle dièdre déterminé par $\theta_{1}, \theta_{2}$ et $2 \varphi_{0}$ (fig. 3 ).

Or, il est évident que l'énergie lumineuse arrivant sur le cristal peut être considérablement augmentée si nous entourons la lampe d'un réflecteur. Un calcul très simple montre qu'il est d'abord nécessaire de placer le réflecteur le plus près possible de la lampe, ce que nous supposerons toujours réalisé.

Le réflecteur cylindrique a donc un diamètre intérieur égal au diamètre extérieur de la lampe.

$\mathrm{Eu}$ égard à nos hypothèses, le résultat précédent est alors multiplié par $(1+\mathscr{R}), \mathscr{R}$ étant le coefficient de réflexion du réflecteur.

Le rendement total de cette configuration est :

$$
\eta=\frac{2 B}{\pi^{2} L}(1+\mathscr{R}) \quad \text { avec } \quad 0<\mathscr{R}<1 .
$$

Il est intéressant d'introduire des grandeurs réduites :

$$
a=r / R \quad m=l / L \quad n=R / L .
$$

Il vient

$$
\begin{aligned}
& \eta=\frac{4(1+\mathscr{R})}{\pi^{2}}\left[a\left\{\sqrt{\frac{1}{4}(1+m)^{2}+n^{2}}-\sqrt{\frac{1}{4}(1-m)^{2}+n^{2}}\right\}\right. \\
& \quad+\frac{a^{3}}{18}\left\{\sqrt{\frac{1}{4}(1+m)^{2}+n^{2}}-\sqrt{\frac{1}{4}(1-m)^{2}+n^{2}+\frac{n^{2}}{\sqrt{\frac{1}{4}(1+m)^{2}+n^{2}}}-\frac{n^{2}}{\sqrt{\frac{1}{4}(1-m)^{2}+n^{2}}}(+\ldots]}\right.
\end{aligned}
$$




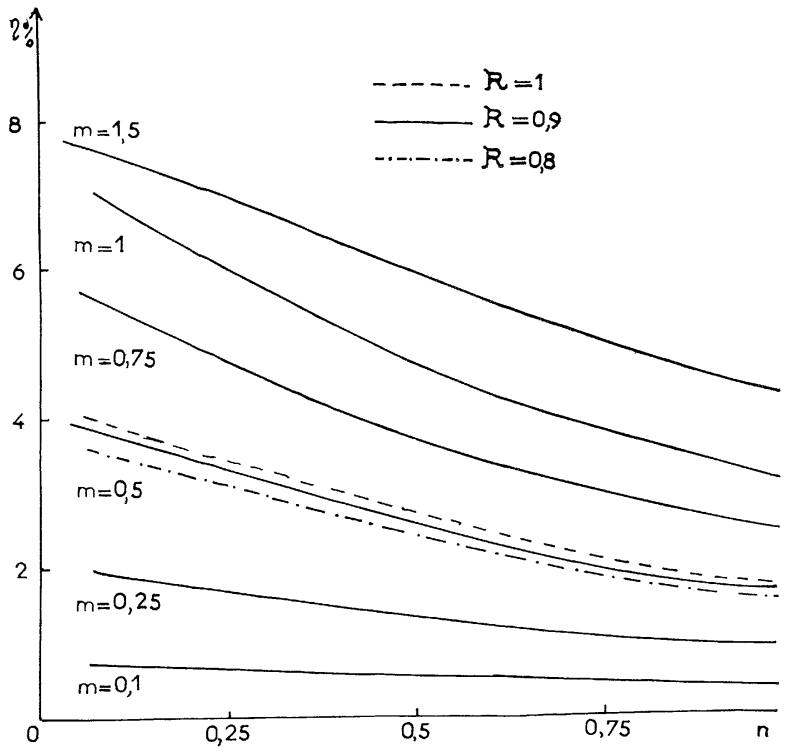

Courbe 1. - Rendement $\eta$ de la configuration hélicoïdale en fonction du rapport $n=R / L$ pour $a=r / R=0,1$ et pour différentes valeurs de $m=l / L$.

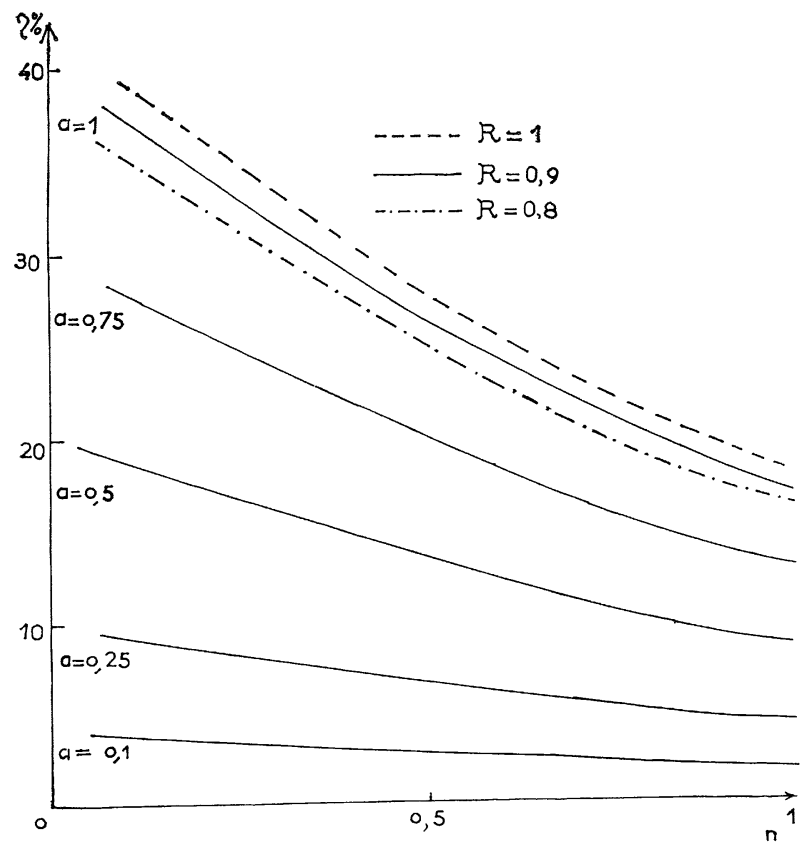

Courbe 2. - Rendement $\eta$ de la configuration hélicoïdale en fonction du rapport $n=R / L$ pour $m=l / L=0,5$ et pour différentes valeurs de $a=r / R$.

ce résultat nous a permis de tracer les courbes (1) $\eta=f(n)$ pour $a$ et $\mathscr{R}$ fixés et pour différentes valeurs de $m$, puis (2) $\eta=f(n)$ pour $m$ fixé et pour différentes valeurs de $a$.

L'examen de ces courbes montre que dans le cas le plus favorable où $m=1(l=L), n=0,25(L=4 R)$, $a=0,75\left(r=\frac{3}{4} R\right)$ et $\mathscr{R}=0,9$, le rendement de la configuration hélicoïdale est voisin de $30 \%$. On ne peut pas espérer obtenir de biens meilleurs résultats car on est alors limité par des considérations mécaniques. En effet, le tube constituant la lampe n'a pas un diamètre nul et on aura au mieux $r=\frac{3}{4} R$.

Le cas où $m$ est supérieur à 1 ne présente que peu ou pas d'intérêt en pratique : le pompage optique du milieu ne serait en effet plus uniforme et l'augmentation de rendement que l'on pourrait espérer obtenir serait contrebalancée par une énergie fournie insuffisante. Toutes choses égales, on peut en effet admettre que l'énergie maximale que peut fournir un flash varie comme sa longueur.

III. Flashes linéaires et réflecteurs elliptiques. 1. Position du PROBLÈme. - Un cristal cylindrique de longueur $l$ et de rayon $r$ est situé sur un des axes focaux d'un cylindre elliptique, et une lampe linéaire de longueur $L$ et de rayon $R$ est située sur l'autre axe focal ( fig. 4).

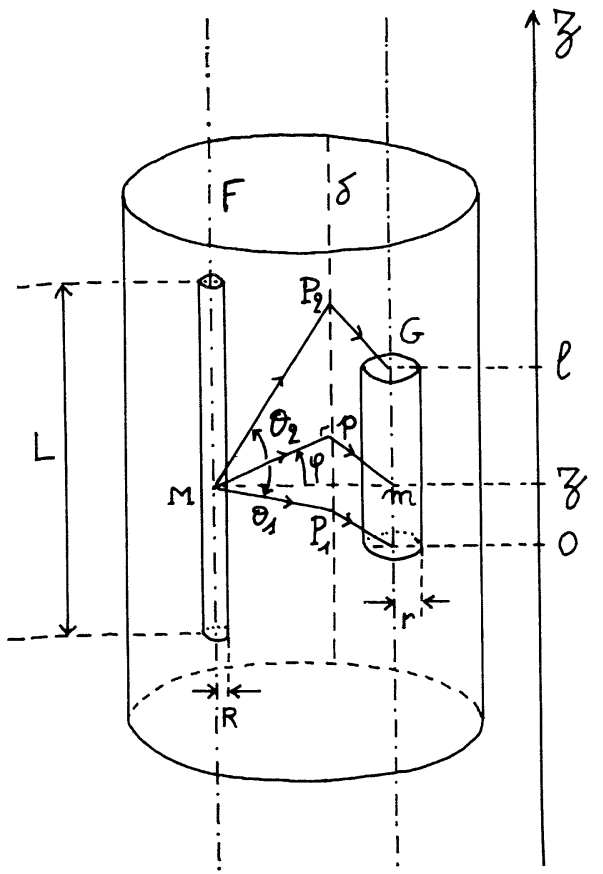

FIG. 4. - Schéma de la configuration elliptique.

Nous calculons le rendement de cette configuration en conservant les hypothèses de la $1^{\text {re }}$ partie. Le cylindre elliptique est un réflecteur dont nous supposons la hauteur du moins égale à $L$.

2. RÉSOlution DU PROBlème. - Nous simplifierons le problème en projetant sur les deux plans perpendiculaires au plan contenant les axes focaux les rayons lumineux issus de la lampe. L'un de ces plans contient $\delta$ 
et est perpendiculaire au plan contenant les axes focaux (fig. 5), l'autre est le plan de section principale (fig. 6).

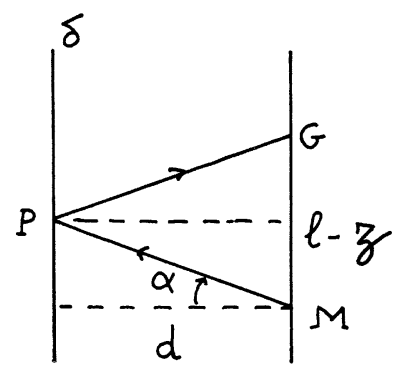

FIG. 5. - Projection des rayons lumineux de M sur un plan contenant $\delta$ et perpendiculaire au plan contenant les axes focaux.

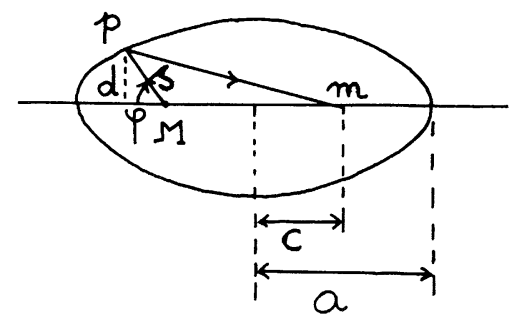

FIG. 6. - Projection des rayons lumineux issus de M sur le plan de section principale du cylindre.

Considérant le point source $M$, l'angle $\alpha \max$, pour lequel l'image de $\mathrm{M}$ se fait en $\mathrm{G}$, point de cote la plus élevée du cristal, est donné par la relation :

soit dans l'espace

$$
\operatorname{tg} \alpha_{\mathrm{M}}=(l-z) / 2 d
$$

$$
\operatorname{tg} \theta_{\mathrm{M}}=(l-z) / 2 s .
$$

Si $e$ est l'excentricité de l'ellipse : $e=c / a$

$$
s=a\left(1-e^{2}\right) /(1+e \cos \varphi) .
$$

Il existe deux angles limites correspondant à une émission au-dessus ou en dessous du plan contenant $\mathbf{M}$ et donnés par :

$$
\begin{aligned}
& \operatorname{tg} \theta_{2 \mathrm{M}}=(l-z) \frac{1+e \cos \varphi}{2 a\left(1-e^{2}\right)}=A(l-z) \\
& \operatorname{tg} \theta_{1 \mathrm{M}}=z \frac{1+e \cos \varphi}{2 a\left(1-e^{2}\right)}=A z .
\end{aligned}
$$

Nous reprenons les mêmes notations que dans la première partie (fig. 4)

$$
\begin{gathered}
\mathrm{d} \Omega=\cos \theta \mathrm{d} \theta \mathrm{d} \varphi \\
I=I_{0} \cos \theta=h \cos \theta \mathrm{d} z \quad h=\mathrm{Cte} \\
\mathrm{d} \Phi=h \cos ^{2} \theta \mathrm{d} \theta \mathrm{d} \varphi \mathrm{d} z \\
\Phi=h \int_{0}^{2 \pi} \mathrm{d} \varphi \int_{\frac{l-L}{2}}^{\frac{l+L}{2}} \mathrm{~d} z \int_{-\theta_{1} \mathrm{M}}^{\theta_{2 M}} \cos ^{2} \theta \mathrm{d} \theta .
\end{gathered}
$$

L'intégration sur $\theta$ se fait immédiatement ainsi que celle sur $z$.
$\Phi=h \int_{0}^{2 \pi}\left[\left(\frac{L+l}{2}\right) \operatorname{arctg} A\left(\frac{L+l}{2}\right)\right.$

$$
\left.-\left(\frac{L-l}{2}\right) \operatorname{arctg} A\left(\frac{L-l}{2}\right)\right] \mathrm{d} \varphi
$$

avec $A=(1+e \cos \varphi) / 2 a\left(1-e^{2}\right)$.

$$
\text { Posons }(L+l) / 2=m \text { et }(L-l) / 2=n \text {. }
$$

$\Phi=h \int_{0}^{2 \pi} m \operatorname{arctg}\left(m \frac{1+e \cos \varphi}{2 a\left[1-e^{2}\right]}\right) \mathrm{d} \varphi$

$$
-h \int_{0}^{2 \pi} n \operatorname{arctg}\left(n \frac{1+e \cos \varphi}{2 a\left[1-e^{2}\right]}\right) \mathrm{d} \varphi
$$

cette intégrale n'est pas calculable de manière analytique, nous avons donc effectué une intégration graphique.

Nous passons de l'expression du flux $\Phi$ à l'expression du rendement $\eta$ par : $\eta=\Phi / \Phi_{\text {total }}$.

Or

$$
\Phi_{\text {tot }}=h \pi^{2} L \text {. }
$$

\section{Soit}

$$
\begin{aligned}
\eta=\frac{1}{\pi^{2} L}\left[\int_{0}^{2 \pi} m\right. & \operatorname{arctg}\left(m \frac{1+e \cos \varphi}{2 a\left[1-e^{2}\right]}\right) \mathrm{d} \varphi \\
& \left.-\int_{0}^{2 \pi} n \operatorname{arctg}\left(n \frac{1+e \cos \varphi}{2 a\left[1-e^{2}\right]}\right) \mathrm{d} \varphi\right] .
\end{aligned}
$$

Nous avons traduit les résultats sous forme d'une famille de courbes. Pour $e$ et $a$ donnés, c'est-à-dire en nous fixant les caractéristiques du réflecteur, nous obtenons (3) $\eta=f(L)$ ipour différentes valeurs de $l$.

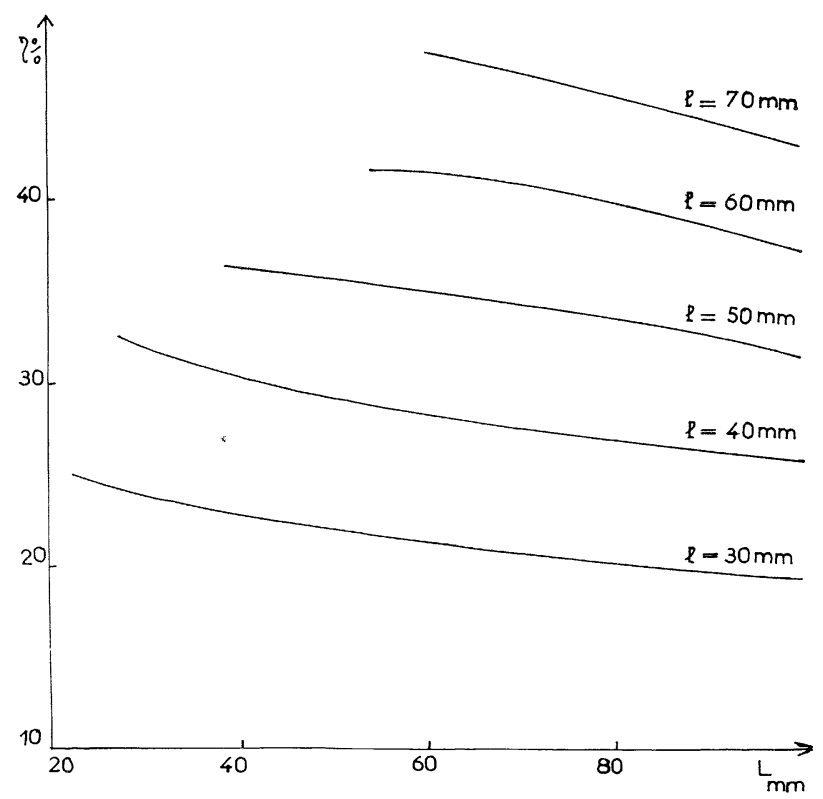

Courbe 3. - Rendement $\eta$ de la configuration elliptique en fonction de la longueur $L$ de la source pour différentes valeurs de la longueur $l$ du récepteur.

Excentricité de l'ellipse : $e=0,2$.

Demi-grand axe de l'ellipse : $a=40 \mathrm{~mm}$. 


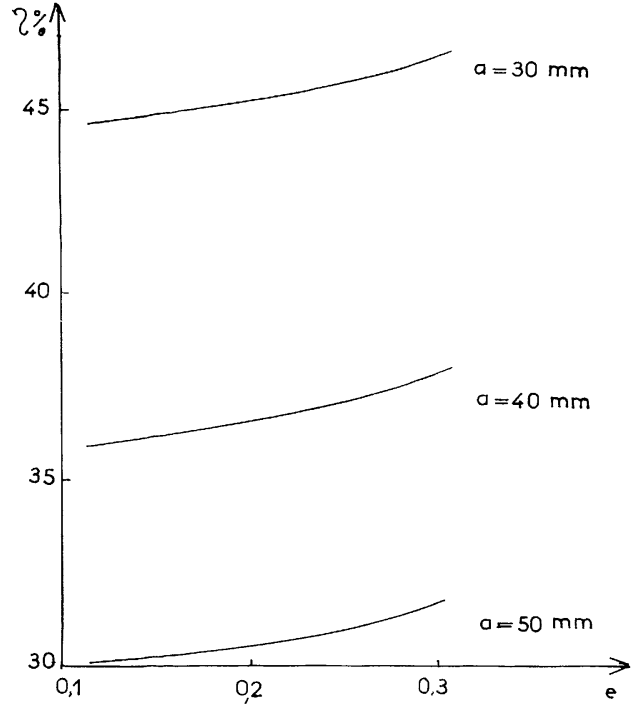

Courbe 4. - Rendement $\eta$ de la configuration elliptique en fonction de l'excentricité $e$ de l'ellipse pour $L=l=$ $50 \mathrm{~mm}$ et pour différentes valeurs de son grand axe $a$.

La deuxième famille de courbes (4) donne $\eta=f(e)$ pour $L=l$ mais pour différentes valeurs de $a$.

Tout ce qui précède ne tient pas compte des diamètres non négligeables de la lampe et du cristal.

On doit en effet tenir compte du fait que l'émission ne se fait pas à partir d'un point mais à partir d'une surface (fig. 7). La section du cristal étant elle aussi définie, on doit calculer la fraction de l'énergie lumineuse captée par le cristal compte tenu de la propriété focalisatrice d'une ellipse et de la loi de Lambert dans le plan.

Les calculs relatifs à cette correction ont été faits par Schuldt et Aagard [3], Ackerman [4] et Bow-

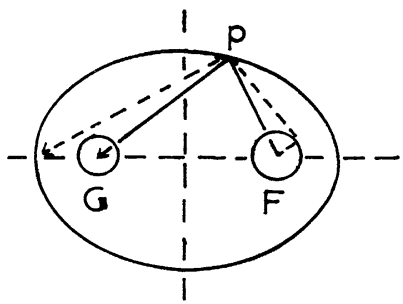

FIG. 7. - L'émission de lumière se fait à partir d'une surface.

ness [5] et donnent, tous les trois, des résultats à peu près analogues.

Cette correction fait intervenir $e$ et le rapport $t=r / R$. Soit $\eta^{\prime}$ le rendement ainsi obtenu.

Le rendement global de la configuration elliptique est alors $\eta \times \eta^{\prime}$ car les deux corrections sont indépendantes.

L'examen des courbes montre alors que pour $e=0,2 \quad a=40 \mathrm{~mm}$ (c'est le cas d'une des ellipses de nos expériences), $L=l=70 \mathrm{~mm}$ et $r=R \ll a$, le rendement global est voisin de $50 \%$, et il peut encore être augmenté, par exemple en diminuant $a$.

Toutefois, si $a$ devient de l'ordre de grandeur de $r$ et $R$, nous tendons vers la configuration dite « closecoupling » à laquelle les résultats précédents ne s'appliquent plus.

On peut considérer qu'un rendement de $60 \%$ est une limite supérieure pour la configuration elliptique.

IV. Vérification expérimentale. - Nous avons procédé à une vérification expérimentale des résultats obtenus en déterminant, compte tenu du rendement calculé, le seuil d'énergie d'un Laser à Rubis.

Le rubis utilisé avait un diamètre de $8 \mathrm{~mm}$ et une longueur de $50 \mathrm{~mm}$. Les faces étaient recouvertes de

TABLEAU I

RUBis $\quad \varnothing=8 \mathrm{~mm} \quad l=50 \mathrm{~mm}$

Flashes LINÉAIRES

\begin{tabular}{|c|c|c|c|c|}
\hline Type & VQX 87 & VQX 107 & $\begin{array}{l}\text { VQX } 1010 \\
-\end{array}$ & PEK XE 1- \\
\hline Énergie de seuil expérimentale. & $374 \mathrm{~J}$ & $440 \mathrm{~J}$ & $470 \mathrm{~J}$ & $410 \mathrm{~J}$ \\
\hline Rendement calculé $\ldots \ldots \ldots \ldots$ & $35 \%$ & $30 \%$ & $28 \%$ & \\
\hline dement ... & $131 \mathrm{~J}$ & $132 \mathrm{~J}$ & $132 \mathrm{~J}$ & $119 \mathrm{~J}$ \\
\hline
\end{tabular}

Flashes héligoidaux

Type $\ldots \ldots \ldots \ldots \ldots \ldots \ldots \ldots \ldots$

Énergie de seuil expérimentale ........

Rendement calculé ...............

Énergie de seuil compte tenu du rendement

VQX 12S 5A
-
$970 \mathrm{~J}$
$14 \%$
$135 \mathrm{~J}$

VQX 15S 8A

PEK XE $531 / 4 \quad 1 / 4$

$14 \%$
$1100 \mathrm{~J}$
$12 \%$
$132 \mathrm{~J}$

$850 \mathrm{~J}$

$16 \%$

$136 \mathrm{~J}$ 
TABLEAU II

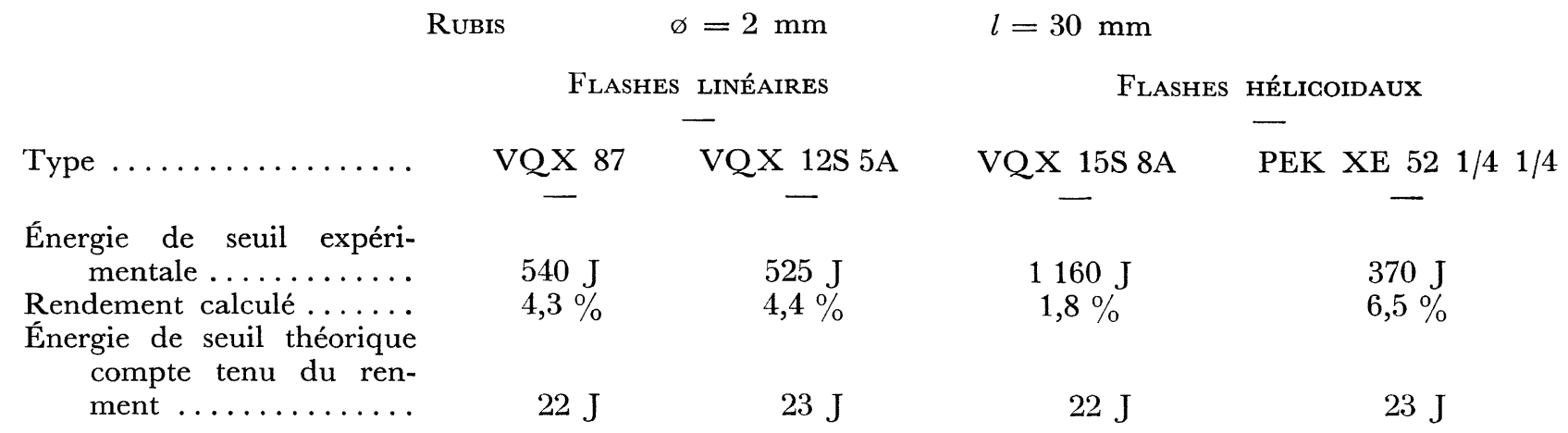

couches multidiélectriques, l'une présentant un facteur de réflexion voisin de $100 \%$ et l'autre de $90 \%$.

Nous utilisons des lampes Flash, pour la plupart de même origine (Verre et Quartz).

Pour les lampes hélicoïdales, le réflecteur était constitué d'une feuille d'aluminium poli. Le réflecteur elliptique était en aluminium ébauché à la fraiseuse et poli [7].

Les caractéristiques du réflecteur elliptique étaient les suivantes :

$$
\begin{gathered}
e=0,25 \text { et } a=40 \mathrm{~mm} \\
\mathscr{R}=0,9 .
\end{gathered}
$$

Un autre rubis de $2 \mathrm{~mm}$ de diamètre et de $30 \mathrm{~mm}$ de longueur nous a donné quelques résultats. Cependant, il était utilisé avec des miroirs extérieurs, ce qui présentait quelques difficultés de manipulation.

Nous avons classé les résultats obtenus dans les tableaux I et II.

V. Conclusion. - L'originalité de ce travail a consisté à tenir compte effectivement de l'émission lambertienne de chaque élément de la lampe dans l'espace. C'est ainsi que nous avons montré que, toutes choses restant égales par ailleurs, le rendement de la configuration elliptique est meilleur que celui de la configuration cylindrique. Un résultat dans ce sens a déjà été obtenu récemment par Kamiryo, Kano, Matsuzawa et Yoshida [6], qui supposaient que l'émission de chaque élément de la lampe n'avait lieu que dans un plan. En pratique, un double problème se pose : celui d'obtenir un seuil le plus bas possible et celui d'utiliser au maximum l'énergie mise en jeu. Le premier problème a été étudié expérimentalement par Congleton et al. [8].

L'ensemble des résultats obtenus de part et d'autre milite pour la validité de la configuration elliptique.

Le couplage entre lampe et cristal peut être encore amélioré par l'introduction de cavités multielliptiques dont le cas, qui n’a pas été traité ici, sera intéressant à étudier.

Manuscrit reçu le 30 septembre 1966.

\section{BIBLIOGRAPHIE}

[1] Ciftan (M.), Luck (C. F.), Shafer (C. F.) et STATZ (H.), "A ruby laser with an elliptic configuration ", Proc. IRE (correspondence), 1961, 49, 960-961.

[2] Tingye Li et Sims (S. D.), Proc. IRE, 1962, p. 464-5.

[3] Schuldi (S. B.) et Aagard (R. L.), Appl. Optics, 1963, 2, no 5, p. 509-513.

[4] ACKERMAN (J. A.), "Optimization of the parameters of multielliptical laser head configurations ", Proc. IRE (correspondence), 1963, 51, pp. 1032-3.
[5] Bowness (C.), Appl. Optics, 1965, 4, no 1, p. 103-107.

[6] Kamiryo (K.), Kano (T.), Matsuzawa (H.), YoSHIDA (M.), Proc. IEEE, 1965, 11, no 53, p. 1750-1.

[7] RaOuli (G.) et FARigoule (F.), J. Physique, 1965, 26, p. $82 \mathrm{~A}-83 \mathrm{~A}$.

[8] Congleton (R. S.), Sooy (W. R.), Dew HIRST (D. R.), RILEY (L. D.), "Comparison of excitation geometries for ruby lasers ». Comptes rendus de la $3^{\text {e }}$ Conférence internationale d'Électronique quantique, p. 1415-1425. 